\title{
Preface: Eli Sercarz Memorial Issue, Part III
}

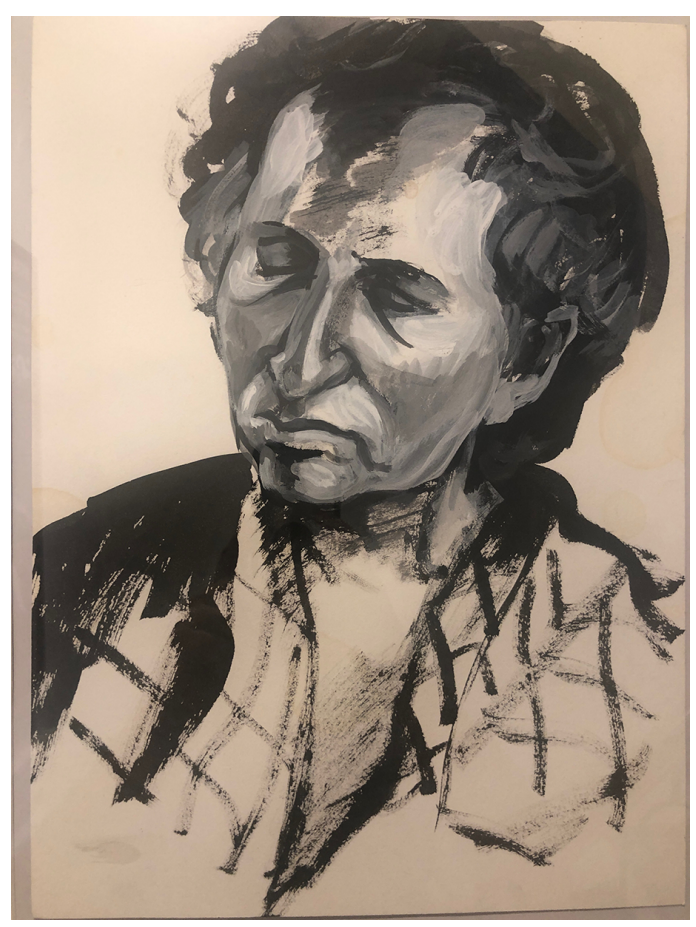

The late Eli E. Sercarz devoted his life and career to the field of immunology, and he systematically dissected several of the unknown fundamental principles and molecular mechanisms that govern the complex immune response mediated by immune cells (T, B, APC). His research investigations and approaches were novel and creative, delineating the means by which specific amino acid sequences of protein molecules dictate a response (immunogenic) or nonresponsiveness (tolerogen) via sophisticated interactions among the APC, T lymphocyte subsets, and B cells. Such meticulous analyses using various protein molecules as models resulted in clarifications of the observed immune defects involved in various human diseases (autoimmune, transplantation, etc.). Eli's guidance and traineeships emanated in hundreds of publications and nurtured the education and development of a large number of academic immunologists, whose careers and research investigations were the direct extensions of his fundamental research.
Eli died at the age of 75 after battling renal cancer. This is the first publication celebrating his memory and legacy. Vipin Kumar and Benjamin Bonavida, guest editors for these special issues in Critical Reviews $^{\mathrm{TM}}$ in Immunology (CRI), were previously Eli's fellows and decided to dedicate three issues in his memory. As anticipated, many of Eli's former fellows and collaborators have responded enthusiastically about dedicating their work in these three issues of CRI, published simultaneously in his memory.

In the third CRI issue, there are eleven contributions, outlined briefly as follows:

Green's article titled "Dances with Cells" reviews the dynamic changes that take place in the immune system throughout life, from birth through death. Green uses the metaphor of Eli's love for dance to explore the life and death of cells in the immune system. He discusses the asymmetric divisions of the activated, naïve CD8T cells, cMyc, and polyamines that play pivotal roles in the lymphocyte functions in aging. He also discusses the consequences of lymphocyte cell death and their clearance.

Riffelmacher and Kronenberg's article entitled "Metabolic Triggers of iNKT Cell Activation during Sterile Auto-inflammatory Disease" reviews the important topic of the mechanisms of the activation of innate-like T lymphocytes: type I or invariant natural killer T cells referred to as iNKT cells. These cells are activated by endogenous ligands and/or microbial antigens from the commensal flora. The iNKT cells, when activated by an inflammatory response, participate in auto-immune responses. For instance, this response is a consequence of obesity-associated inflammation and fatty liver disease, as well as ischemia-reperfusion injuries and sickle cell disease. Inflammation in the intestine shapes the iNKT responses through the microbiome. Riffelmacher and Kronenberg review the common themes of the molecular pathways that cause iNKT cell autoreactivity and the quality of iNKT cell responses.

Coelho et al.'s article entitled "Towards an Integrated View of Operational Tolerance in Human 
Renal Transplantation: A Systems Biology Perspective" reviews the concept of "operational tolerance" (OT), in which the body does not reject human renal and liver transplantations following the discontinuation of immunosuppression. Coelho and collaborators utilized the Brazilian Multicenter Study on OT and integrated original data on systems biology in OT and identified underlying mechanisms. They investigated dominant discriminating molecules in OT compared to chronic rejection (CR). Overall, Coelho and collaborators' initial investigation of systems biology in OT in human renal transplantation underscored the richness of combining different strategies to search for potentially dominant mechanisms. This allowed for building molecular networks to identify interconnections among potential mechanisms. In addition, these analyses provide novel potential therapeutic targets to harness immunoregulation and build and preserve homeostasis in human transplantation.

Zaghouani and Miller's article entitled "Early-Life Th1 Immunity" reviews the findings that while newborn mice exposed to an antigen at birth are rendered tolerant, they respond to a challenge by the same antigen later in life. The secondary responses exhibited a skewing of response toward Th2 cells. Zaghouani and Miller review the mechanisms underlying the deficit of neonatal Th1 cells and associate these findings with the poor efficacy of neonate vaccinations. The mechanism behind poor efficacy of neonatal vaccination can be tied to HR-mediated Th1 apoptosis. They suggest that vaccine formulations that can preserve Th1 cells will potentiate the vaccine efficacy in neonates.

Franco's article entitled "Immune Regulation Is the Most Relevant Arm of the Immune Response in Infants and Young Children" reviews immunity in infants and how it differs from adults. Both the innate and adaptive immune cells' repertoires in babies are reviewed and found to be different from those of the adults. Franco concludes that immunity in infants is shifted toward the immune tolerance underlying the roles of the tolerogenic myeloid dendritic cells (tmDCs) and the Treg cells with a thymic-derived phenotype.

Franco's memorial "Me and Eli" discusses his close relationship with Eli, as his fellow, and how grateful and professionally lucky he is to have been associated with him. He states: "I hope that all of us will keep the legacy of Eli's intellectual independence, fairness, curiosity, love for life, and love for science with profound reciprocal respect."

Kalil and Guilherme's article entitled "Rheumatic Fever: A Model of Autoimmune Disease Due to Molecular Mimicry between Human and Pathogen Proteins" reviews the role of the immune system in acute rheumatic fever (ARF) and its major sequela rheumatic heart disease (RHD). Both the humoral and cell-mediated immune responses are involved in RHD, and Kalil and Guilherme review the underlying immune mechanisms responsible for triggering RHD. They discuss the data that demonstrate "the development of autoimmune reactions, due to a previous bacterial infection, that cause lesions in the heart valves, leading to heart damage and poor quality of life in young and adolescent individuals."

Nanda's article entitled "Tissue Resident Memory T Cells: Sheltering-in-Place for Host Defense" reviews the new paradigm of the presence of noncirculatory, tissue-resident memory T cells (TRM) that are key in protection against reinfections, tissue surveillance, cancer, and autoimmune and allergic diseases. These TRM cells contrast with the previous view that T circulatory memory cells (TcirM) are the main defenders against reinfections and other diseases. Tissue-lodged TRM oftentimes can mediate host defense, completely autonomous of circulating T cells. Quoting from Nanda, "Since TRM are recognized to be the main actors in the first line of adaptive defense against recrudescent pathogens, the target of vaccine efforts must also shift to seeding and activation of trigger-ready TRM cells within the organ or tissue of pathogen invasion. In parallel, therapies for relapsing autoimmunity must be redirected toward inactivating the locally anchored, TRM cell responses."

Haskins and Wenzlau's article entitled "CD4 T Cells and Neo-Antigens in Autoimmune Diabetes" reviews key findings related to the isolation of diabetogenic CD4 $\mathrm{T}$ cell clones mediating type 1 diabetes (TID) in the NOD mouse model. These CD4 T cell clones induce rapid disease and overt hyperglycemia in both the young NOD mice and in the NOD/ 
SCID mice lacking $T$ cells. The use of these clones was significant to investigate their diabetogenic role and to identify neoantigens that they recognize. These investigators have successfully generated $\mathrm{T}$ cell receptor transgenic (TCR-Tg) mice that proved to be an excellent resource for the study of mechanisms of TID, both in the authors' laboratories and in other laboratories.

Haskins's memorial "Remembering Eli Sercarz" reflects on the beginnings of her very long collaboration with Eli over the years.

Scheherazade Sadegh-Nasseri's article, entitled "How a Proposed Hypothesis during My PhD Training Shaped My Career" briefly details how Eli's mentorship sets Sadegh-Nasseri's quest to address some of the key questions in her laboratory related to peptide-MHC binding, immunodominance, $\mathrm{T}$ cell recognition and $\mathrm{T}$ cell differentiation. After outlining many important contributions and terminologies from Eli's laboratory, she describes the molecular basis of antigen presentation, involvement of DM/DO and CLIP in epitope selection, and how differences in antigen presentation result in differential signaling, ultimately leading to altered
$\mathrm{T}$ cell activation and anergy. Immunodominance involves several factors, including determinant capture, MHC-binding, availability and determinant hierarchy. She discusses how anti-specific B cells play a crucial role under conditions of low antigen load and for memory $\mathrm{T}$ cell activation and how these interactions could be important for the selection of high affinity CD4 $\mathrm{T}$ cells.

The provided reviews discuss up-to-date studies on various immune system topics, several of which resulted from expansions of original findings from Eli's laboratory. The contents of this CRI issue also emphasize the many outcomes of Eli's significant conceptual contributions for managing human diseases today.

Guest Editors:

Vipin Kumar

University of California San Diego

La Jolla, CA, USA

Benjamin Bonavida

University of California, Los Angeles

Los Angeles, CA, USA 